\title{
ARTICLE The JAK inhibitor ruxolitinib reduces inflammation in an ILC3-independent model of innate immune colitis
}

\author{
A. M. Overstreet ${ }^{1}$, D. L. LaTorre ${ }^{2}$, L. Abernathy-Close ${ }^{1}$, S. F. Murphy ${ }^{3}$, L. Rhee ${ }^{3}$, A. M. Boger ${ }^{1}$, K. R. Adlaka ${ }^{2}$, A. M. Iverson ${ }^{2}$, D. S. Bakke ${ }^{3}$, \\ C. R. Weber ${ }^{4}$ and D. L. Boone ${ }^{1,2}$
}

Innate immunity contributes to the pathogenesis of inflammatory bowel disease (IBD). However, the mechanisms of IBD mediated by innate immunity are incompletely understood and there are limited models of spontaneous innate immune colitis to address this question. Here we describe a new robust model of colitis occurring in the absence of adaptive immunity. RAG1-deficient mice expressing TNFAIP3 in intestinal epithelial cells (TRAG mice) spontaneously developed 100\% penetrant, early-onset colitis that was limited to the colon and dependent on intestinal microbes but was not transmissible to co-housed littermates. TRAG colitis was associated with increased mucosal numbers of innate lymphoid cells (ILCs) and depletion of ILC prevented colitis in TRAG mice. ILC depletion also therapeutically reversed established colitis in TRAG mice. The colitis in TRAG mice was not prevented by interbreeding to mice lacking group 3 ILC nor by depletion of TNF. Treatment with the JAK inhibitor ruxolitinib ameliorated colitis in TRAG mice. This new model of colitis, with its predictable onset and colon-specific inflammation, will have direct utility in developing a more complete understanding of innate immune mechanisms that can contribute to colitis and in pre-clinical studies for effects of therapeutic agents on innate immune-mediated IBD.

Mucosal Immunology (2018) 11:1454-1465; https://doi.org/10.1038/s41385-018-0051-2

\section{INTRODUCTION}

Ulcerative colitis and Crohn's disease are chronic debilitating inflammatory disorders of the gastrointestinal tract. The etiology of these inflammatory bowel diseases (IBDs) is not known but the pathology includes excessive activation of the immune system leading to damage of the intestinal mucosa and loss of normal function. The innate immune system has a key role in this inflammation, as it is the first to react to microbial challenges, directs the adaptive immune response, and generates the final cellular effectors of immune-mediated inflammation. In most models of spontaneous genetically driven IBD, adaptive immune cells, in particular T cells, are essential for colitis. ${ }^{1}$ However, the discovery of a parallel network of innate lymphoid cells (ILCs) enriched at mucosal sites and capable of producing cytokines comparable to T-helper cells, suggests that the innate immune system alone may be capable of initiating and sustaining colitis independently from adaptive immune cells. ${ }^{2-4} \mathrm{~A}$ better understanding of how innate immune-mediated inflammation contributes to IBD is needed in order to identify promising therapies to treat these diseases. Here we describe a new robust model of spontaneous innate immune mediated colitis that will facilitate a better understanding of innate immune contributions to IBD.

TNFAIP3, also known as A20, is a cytosolic ubiquitin-editing enzyme that inhibits innate immune receptor signaling and tumor necrosis factor (TNF)-induced cell death. ${ }^{5-8}$ TNFAIP3-associated genetic variants have been implicated in human IBD and other inflammatory diseases. ${ }^{8,9}$ Mice lacking TNFAIP3 develop inflammation, as they are unable to properly inhibit microbial activation of innate receptors. ${ }^{5,} 10$ This is compounded by the fact that TNFAIP3 $^{-1-}$ mice also cannot control TNF-induced inflammation and are susceptible to TNF-induced cell death. ${ }^{7}$ Cell-type-specific roles for TNFAIP3 in intestinal inflammation have been demonstrated by lineage-specific deletions showing that TNFAIP3 mainly suppresses inflammation in myeloid cells, while it mainly suppresses cell death in intestinal epithelial cells (IECs). ${ }^{11}$ Expression of TNFAIP3 in IEC improves barrier function and protects against DSS colitis and IEC death. ${ }^{12,}{ }^{13}$ However, expression of TNFAIP3 in IEC results in invasion of the colonic inner mucus layer by bacteria and increased susceptibility to colitis in IL10 $0^{-1-}$ mice. ${ }^{14}$ Thus, the role of TNFAIP3 in intestinal inflammation is both cell and context dependent. Here we describe a new model of IBD wherein immunodeficient RAG $1^{-1-}$ mice that express TNFAIP3 in IEC spontaneously develop $100 \%$ penetrant early-onset colitis.

This new model will allow us to better understand the role of innate immunity in colitis and test the effects of established and emerging therapies for IBD. In this study, we show that ILC depletion can prevent and can also reverse established colitis, suggesting that ILC targeted therapies may be able to maintain and induce IBD remission, respectively. ILCs act through production of cytokines that can induce responses in target cells through JAK-STAT signals. JAK Inhibitors have shown promise in clinical trials for IBD and genetic variants associated with JAK2 increase risk for IBD. ${ }^{15,16}$ In vitro studies have shown both increased and decreased innate immune inflammation in response to JAK inhibitors. $^{17-20}$ Thus, in vivo models of innate immune

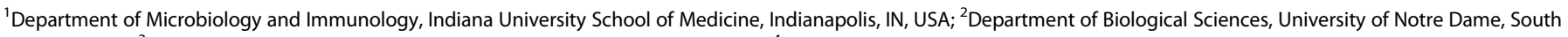
Bend, IN, USA; ${ }^{3}$ Department of Medicine, University of Chicago, Chicago, IL, USA and ${ }^{4}$ Department of Pathology, University of Chicago, Chicago, IL, USA

Correspondence: D L. Boone (daboone@iu.edu)

These authors contributed equally: A. M. Overstreet, D. L. LaTorre, L. Abernathy-Close.

Received: 28 July 2017 Revised: 23 May 2018 Accepted: 31 May 2018

Published online: 9 July 2018 
a

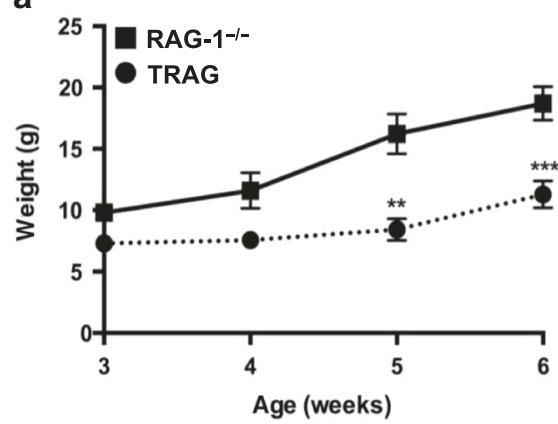

b

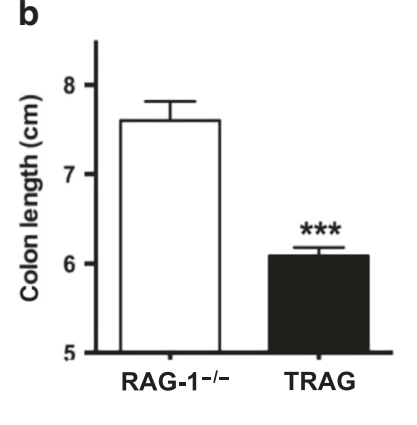

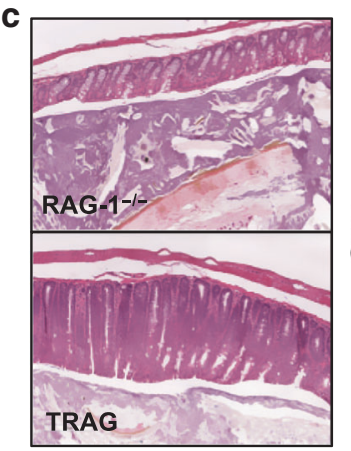

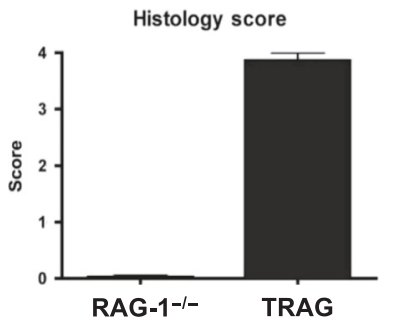

Fig. 1 Spontaneous innate immune mediated colitis in TRAG mice. Mice were monitored for health and the onset of colitis by (a) body weight vs. age, and following euthanasia at 6 weeks of age for (b) colon length and (c) histology. ${ }^{* *} p<0.01,{ }^{* *} p<0.005$ ( $n>5$ mice/group)

inflammation are necessary to help resolve these inconsistencies. In this study we find that ruxolitinib, a JAK1/JAK2 inhibitor, suppresses innate immune-mediated intestinal inflammation. This new robust model of innate immune colitis will provide important insight into innate mechanisms of IBD and allow testing of therapies for their effects on innate immune inflammation in vivo.

\section{RESULTS}

Spontaneous innate immune-mediated colitis

We previously demonstrated that villin-TNFAIP3 $\times \mathrm{IL}^{-1} 0^{-1-}$ mice develop early-onset $100 \%$ penetrant colitis, which was not observed in villin-TNFAIP3 or IL $10^{-1-}$ mice (all on a C57BI/6 background). ${ }^{14}$ To investigate the role of regulatory $T$ cells in this model, we crossed villin-TNFAIP3 mice to immunodeficient RAG $1^{-\prime-}$ mice with the intention of studying the adoptive $T$ cell transfer model of colitis in these mice. However, villin-TNFAIP3 $x$ RAG $1^{-1-}$ mice (TRAG mice) spontaneously developed $100 \%$ penetrant colitis by the time they were 5-6 weeks of age. This was evident by reduced body weight, shorter colon length and histology showing extensive colitis in TRAG mice (Fig. 1). This inflammation was not observed in villin-TNFAIP3 or in RAG1 ${ }^{-/-}$ mice co-housed as littermates with TRAG mice. Mouse breeding and studies shown in Fig. 1 were undertaken at the University of Chicago animal care facility but all subsequent work has been performed in the animal care facilities at the University of Notre Dame and this phenotype has persisted across both institutions. Thus, epithelial expression of TNFAIP3 combined with immunodeficiency results in spontaneous, early onset, 100\% penetrant, innate immune-mediated colitis.

Innate immune cells are increased in TRAG colitis

To evaluate the immune cells contributing to colitis in TRAG mice, we collected lamina propria leukocytes (LPLs) from co-housed 4and 8-week-old TRAG and RAG $1^{-1}$ littermates. The mucosa of TRAG mice contained increased numbers of $\mathrm{CD} 45^{+}$leukocytes including neutrophils, inflammatory monocytes (Ly6C ${ }^{\text {hi }}$ ) and ILCs (Fig. 2). The increase in mucosal innate cells was evident as early as 4 weeks of age, prior to overt clinical signs of colitis in TRAG mice. Thus, colitis in TRAG mice is characterized by increased numbers of innate immune cells, including ILC, in the colonic mucosa.

Gene and cytokine expression in TRAG mice

In order to understand the nature of colitis in this model, we analyzed gene expression in the colon, comparing TRAG vs. $\mathrm{RAG}^{-/-}$mice after the establishment of colitis in TRAG mice. Principle component analysis revealed differential gene expression in TRAG vs. RAG1 ${ }^{-/-}$mice. RNASeq profiles revealed differential expression of 6663 genes in TRAG Vs. RAG1 ${ }^{-1-}$ colonic tissue. These included 174 genes with greater than log2fold change increase and 102 genes with more than log2fold decreased expression in TRAG vs. RAG1 ${ }^{-/-}$colons. Pathway analysis was consistent with interferon- $\gamma$ (IFN $\gamma$ ), interleukin (IL)-22 and IL17 gene expression signatures, as well as activation of JAK-STAT signaling in TRAG colons, suggestive of cytokine-mediated inflammation (Fig. 3). In addition, increased expression of chemokines and a signature suggestive of neutrophil and myeloid cell recruitment/ migration/chemotaxis was evident in TRAG colons. This is consistent with the increased numbers of neutrophils and inflammatory myeloid cells we observed in the mucosa of TRAG mice. The increased expression of IFNY and IL-22, along with genes induced by these cytokines, was confirmed by quantitative PCR (Fig. 3). To assess cytokine secretion from mucosal immune cells, we cultured LPLs in media and measured cytokine production using a flow cytometric multi-plex bead based assay. Supernatants of LPLs from TRAG mucosa produced increased amounts of IFNY, TNF, IL-22, IL-5, IL-1 $\beta$, and IL-9, compared with those from RAG $1^{-1-}$ mice (Fig. 3 ). In the absence of adaptive immune cells, the cytokine profile of the colitic mucosa of TRAG mice is consistent with activation or increased numbers of ILC (IFNY, TNF, IL-5, IL-9, and IL-22) or other cytokine producing cells such as inflammatory macrophages (IL-1 $\beta$ ).

\section{ILC depletion prevents colitis in TRAG mice}

The results showing increased numbers of ILC and a cytokine profile consistent with ILC activation in the intestinal mucosa of TRAG mice suggested that these cells might be contributing to colitis in this model. To test this, we immunodepleted ILC from TRAG mice beginning at 4 weeks of age using anti-Thy 1 antibody (or pre-immune serum as control) injected intraperitoneally (i.p.) every 3 days for at total of 15 days, as previously described. ${ }^{4}$ TRAG mice treated with control antibody exhibited clinical and histological signs of colitis comparable to untreated TRAG mice (Fig. 4). Conversely, mice treated with antibody to deplete ILC showed signs of clinical improvement and markedly reduced histological signs of inflammation (Fig. 4). In addition, LPLs from TRAG mice treated with anti-Thy 1 antibody displayed reduced numbers of mucosal ILC, neutrophils and inflammatory myeloid cells (Fig. 4). Thus, depletion of ILC prevents innate immune mediated colitis in TRAG mice.

ILC depletion reverses established colitis in TRAG mice One goal of IBD therapy is to induce remission in patients with disease. This can be modeled in mice by allowing colitis to develop before therapeutically treating disease. ILC depletion in young TRAG mice prevents colitis indicating that ILC are involved in the initiation of innate immune colitis. Whether ILC has a role in maintaining established inflammation in colitis is not known. The colitis in TRAG mice is accompanied by persistently elevated numbers of ILC, suggesting that ILC may have a role in maintaining this innate immune colitis (Fig. 2). To test this we 

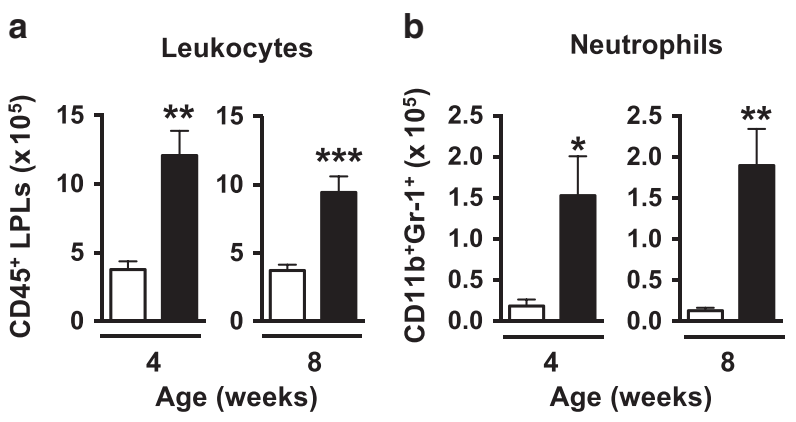
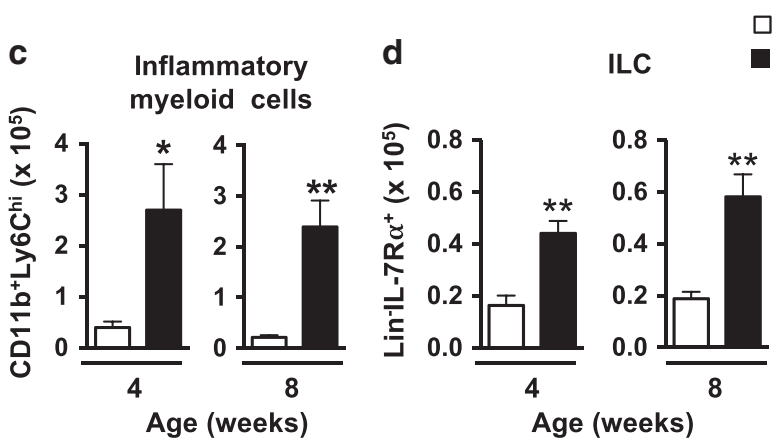

e

4 Weeks old

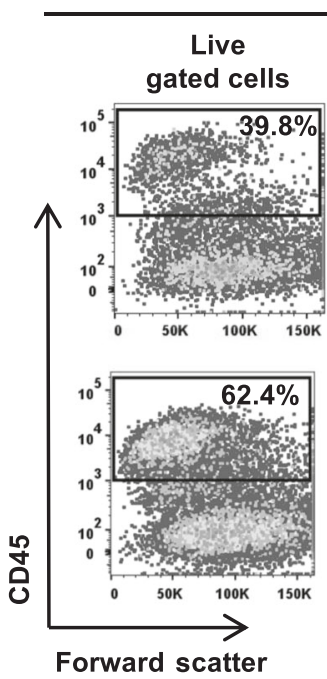

f

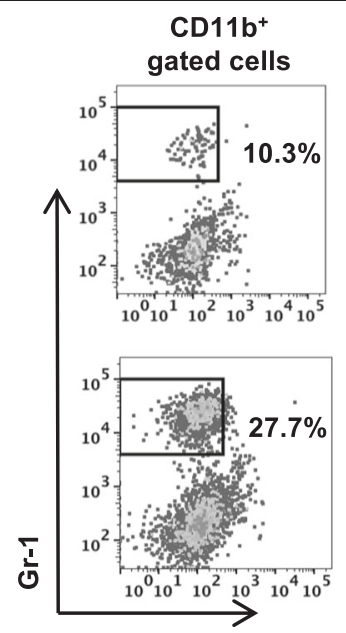

Siglec F

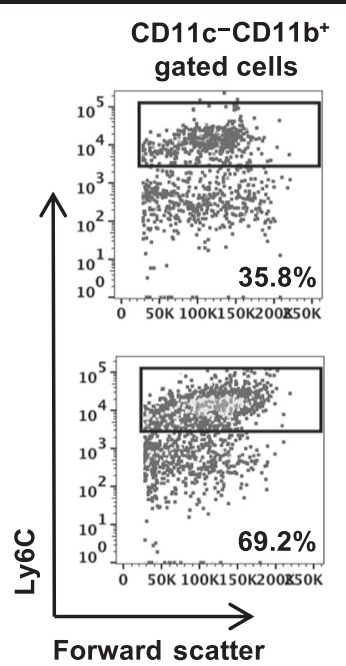

Forward scatter $\square$ RAG

TRAG 
a
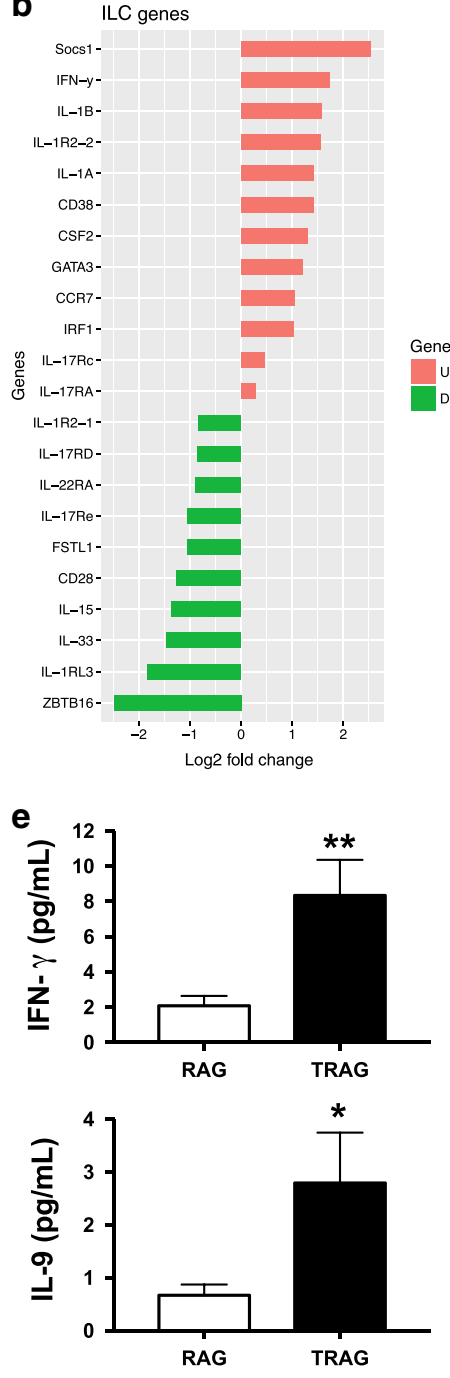

d
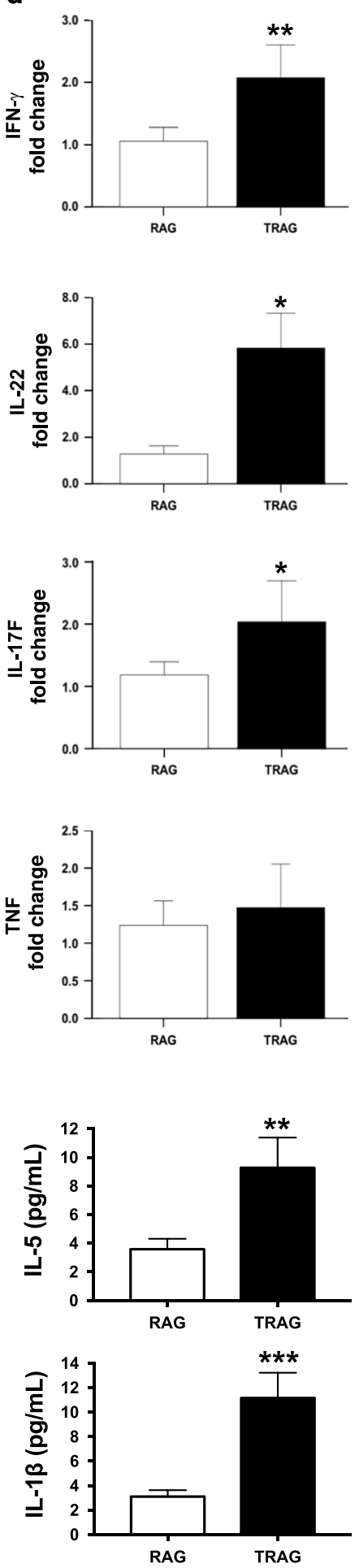

reverse established innate immune colitis to induce remission of disease.

TRAG colitis is distinct from other innate models of IBD Innate immune colitis in T-bet $^{-/-} \times \mathrm{RAG}^{-/-}$mice (TRUC) mice is induced by gut microbes and is transmissible to co-housed RAG $2^{-1-}$ or wild-type (WT) mice. We did not observe any transmission of colitis to co-housed $\mathrm{RAG}^{-1-}, \mathrm{v}$-TNFAIP3, or WT littermates of TRAG mice. Three possible explanations of this are that microbes are not involved in TRAG colitis, or that the microbes involved in TRAG colitis are not transmissible, or that microbial transmission occurs but does not transfer colitis as seen in TRUC mice. To test whether microbes drive colitis in TRAG mice, we administered antibiotics to TRAG mice beginning at weaning for three weeks. Antibiotic treatment ameliorated colitis in TRAG mice, indicating that microbes induce colitis in this model (Fig. 6). 

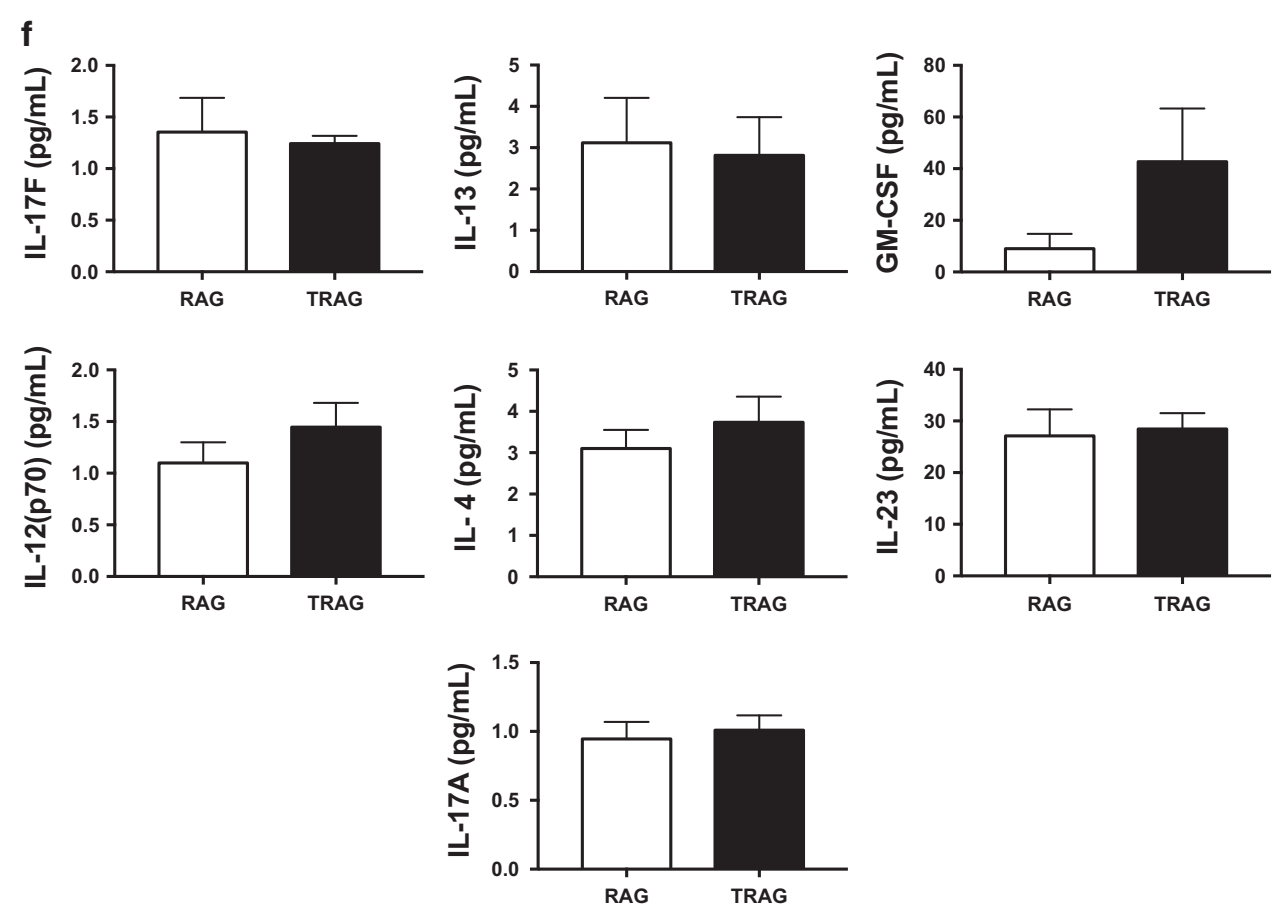

Fig. 3 Gene expression and cytokine profile of TRAG colitis. (a) Principal component analysis of gene expression in TRAG vs. RAG $1^{-1-}$ colonic mucosa. (b, c) Molecular drivers of the differential gene expressions patterns identified using Ingenuity Pathway Analysis of genes associated with colitis in TRAG mice implicates the (b) ILC and (c) JAK1/2 pathway. (d) Quantitative PCR showing increased expression of INF $\gamma$, IL-22, and IL17, but not TNF, in the mucosa of TRAG mice. (e) Cytokine secretion by LPLs isolated from RAG ${ }^{-/-}$vs. TRAG mucosa showing increased secretion of IFN $\gamma$, TNF, IL-5, IL-9, IL-22, and IL-1 $\beta$ from LPLs of TRAG mice. (f) Cytokines detected but not statistically different between TRAG and RAG1 ${ }^{-1-}$ LPLs included IL-17F, IL-17A, GM-CSF, IL-23, IL-4, IL-12, and IL-13. (Mann-Whitney) ${ }^{*} p<0.05,{ }^{* *} p<0.01,{ }^{* * *} p<0.001(n=7-10$ mice/group (except TRAG array group where $n=4)$ )

Thus, although colitis is induced by microbes in both the TRUC and TRAG models, TRAG colitis is distinct from TRUC colitis in that it is not transmissible to co-housed mice.

Colitis can be induced in $\mathrm{RAG}^{-1-}$ mice infected with Helicobacter hepaticus. This innate immune colitis model is accompanied by hepatitis. We did not observe hepatitis in any TRAG mice nor did we observe colitis in any $\mathrm{RAG}^{-1-}$ mice in our studies (Fig. 6). Acute and transient innate colitis can also be induced by administration of anti-CD40 antibody to RAG $^{-1-}$ mice and this is accompanied by systemic inflammation, hepatopathology and splenomegaly. The colitis in TRAG mice was chronic, restricted to the colonic mucosa and accompanied by no signs of inflammation in the terminal ileum, spleen or liver indicating that this model is distinct from the anti-CD40 innate model of IBD (Fig. 6).

Existing models of innate colitis are mediated by group 3 ILC and are thus ameliorated by interbreeding with mice lacking the transcription factor RoRyt. To test for the role of group 3 ILC in our model we crossed TRAG mice to Rorc $^{-1-}$ mice and assessed for colitis. TRAG $\times$ Rorc $^{-1-}$ mice exhibited colitis with comparable incidence as TRAG littermates (Fig. 6). Histologically, there was some evidence of attenuation of colitis severity in TRAG $x$ Rorc $^{-1-}$ mice compared to TRAG littermates, indicating that group 3 ILC3 may contribute to colitis severity in TRAG mice, which is consistent with the increased secretion of IL-22 by TRAG LPLs (Figs. 3, 6). This indicates that the colitis in TRAG mice, unlike other innate models of colitis, can occur independently of RoRyt and is therefore not dependent on group 3 ILC (or ILC derived from plasticity of group 3 ILC).

Colitis in TRUC mice can be ameliorated with anti-TNF antibody treatment. To assess the role of TNF in the TRAG colitis model, we administered anti-TNF antibody to TRAG mice beginning at weaning for 3 weeks. TRAG mice treated with anti-TNF antibody exhibited colitis comparable to TRAG mice (Fig. 6). Thus, unlike the TRUC model of colitis, TRAG colitis is not ameliorated by anti-TNF therapy. Together these findings indicate that the innate immune colitis observed in TRAG mice is distinct from other innate immune models of IBD.

JAK inhibition prevents innate immune mediated colitis ILC respond to and produce cytokines that signal through receptors acting via the JAK/STAT pathway. Our gene array results were consistent with increased JAK/STAT signal induced gene expression. We therefore assessed STAT activation in Thy $1.2^{+}$LPLs isolated from TRAG and RAG $1^{-1-}$ mice. Flow cytometric staining indicated increased intensity of phospho-STAT1 in the Thy $1.2^{+}$ LPLs isolated from the mucosa of TRAG mice compared to RAG1 ${ }^{-1}$

littermates, implicating JAK/STAT signaling in the colitis of TRAG mice (Fig. 7).

JAK inhibitors have shown promise in clinical trials for IBD. ${ }^{15}$ We therefore tested whether JAK inhibition could prevent colitis in TRAG mice. Four-week-old TRAG mice were administered the JAK2/1 inhibitor ruxolitinib (or dimethyl sulfoxide (DMSO) vehicle control) by gavage twice daily from three to seven weeks of age. Ruxolitinib-treated mice displayed improved clinical scores for IBD and this was confirmed at necropsy by gross examination of the colon (Fig. 7). LPL preparations displayed reduced innate immune cell invasion in the mucosa of TRAG mice treated with ruxolitinib. Histology also indicated that treatment with ruxolitinib treatment trended toward reduction of the severity of colitis in TRAG mice (Fig. 7). The effects of ruxolitinib on colitis were muted, because we observed less colitis than typically seen at this age in the control mice that were treated with DMSO (vehicle). We suspect that DMSO alone reduced some of the intestinal inflammation in TRAG mice, as DMSO is known to have anti-inflammatory properties in vivo. ${ }^{21}$ Thus, the innate immune mediated colitis in 
a

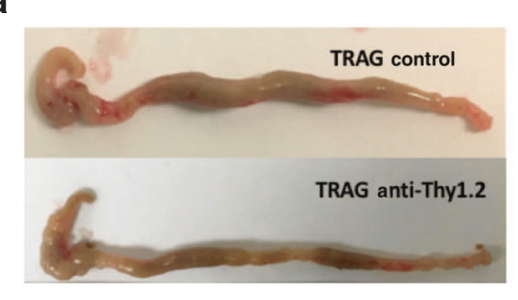

b

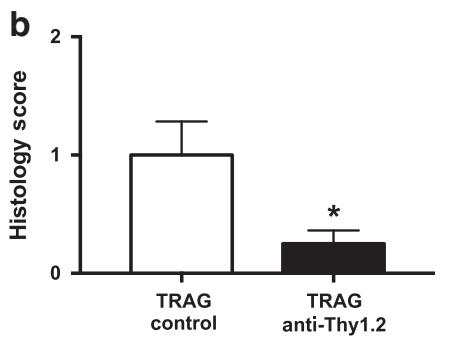

c
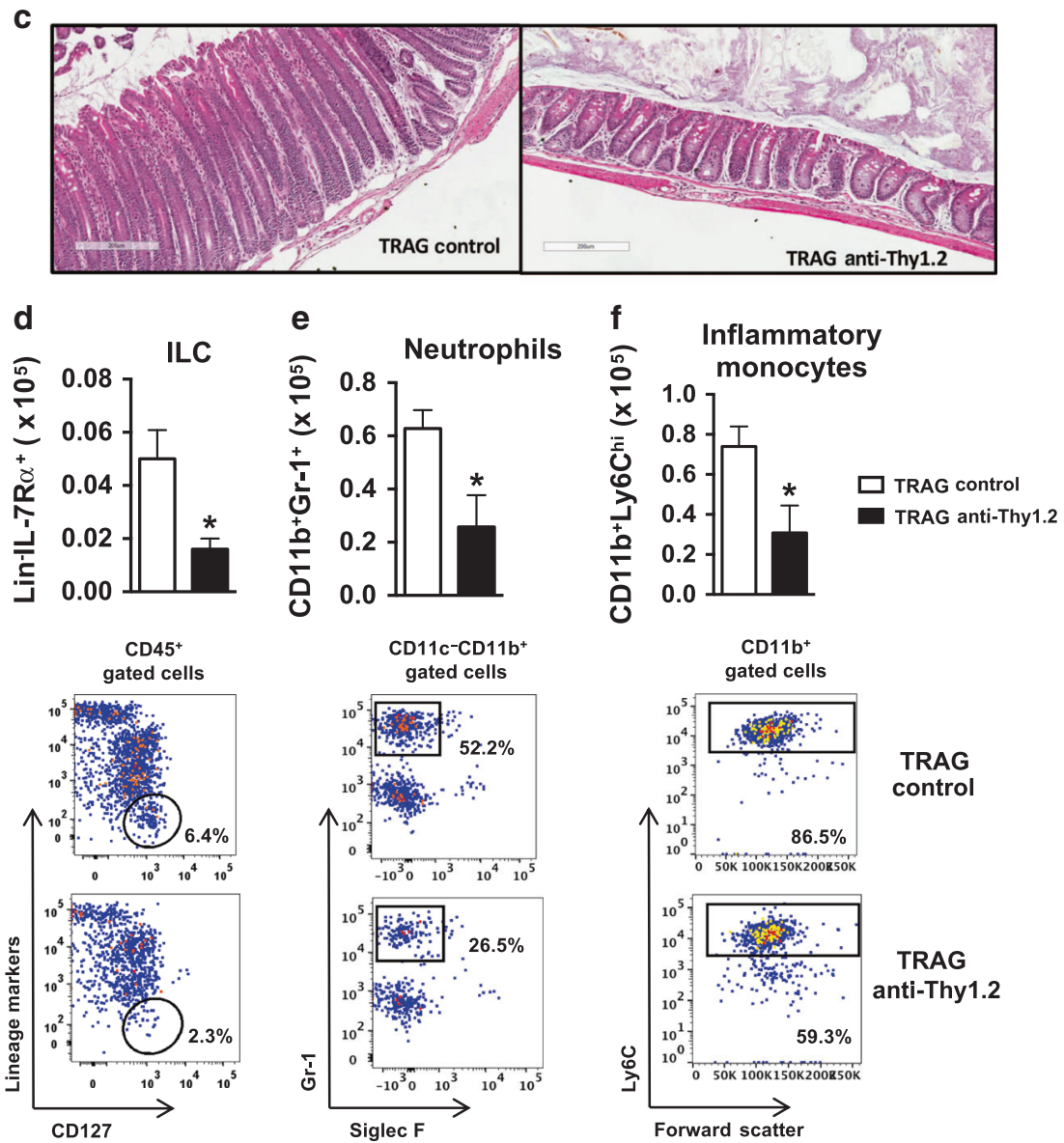

Fig. 4 Prophylactic anti-Thy1.2 reduces ILCs and ameliorates colitis in TRAG mice. IP injections of anti-Thy1.2 (1 mg) were administered to TRAG mice from 4-6 weeks of age every 3 days. Anti-Thy 1.2 treatment reduced colitis in TRAG mice as indicated by (a) colon length and gross morphology and (b, c) histology. TRAG mice treated with anti-Thy 1.2 antibody prior to the development of colitis displayed reduced numbers of colonic mucosal (d) ILC, (e) neutrophils, and (f) inflammatory myeloid cells, compared with control TRAG mice. (Mann-Whitney for (b); T-test for other data) ${ }^{*} p<0.05, p<0.005$. Data represent two independent experiments, mean \pm SEM $(n=8-10$ mice/group)

this model involves increased activation of STAT signaling in Thy $1.2^{+}$cells and can be ameliorated by pharmaceutical inhibition of JAK2/1.

\section{DISCUSSION}

This study is the first to describe the spontaneous innate immune mediated colitis that occurs in TRAG mice. This model will be invaluable for improving our understanding of innate immune inflammation in colitis as it is robust, occurring with $100 \%$ penetrance with an early and predictable age of onset. One advantage of a predictable and early-onset colitis is that it allows for clinical studies that model induction and maintenance of remission that are less tractable in other spontaneous colitis models wherein colitis onset is more stochastic and can occur at much later ages in mice. In the present study, we used this model to demonstrate that depletion of ILC can reverse established colitis and also prevent the establishment of colitis, suggesting that ILC depletion may be able to both induce and maintain remission of innate immune colitis, respectively. The ability to reverse established colitis by depletion of ILC supports prior studies showing that therapeutic treatment of $H$. hepaticus innate colitis with Thy 1.2 antibody reversed intestinal inflammation and colitis associated cancer. ${ }^{22}$ The cytokine IL-22 was identified as pathogenic in that model and we observed elevated IL-22 secretion from LPLs of TRAG mice. In the $H$. hepaticus model, pathogenic IL-22 originated from $\mathrm{Nkp} 46^{-}$ILC, suggesting that ntural killer (NK) cells were not driving the chronic stages of colitis in that model. ${ }^{22}$ One possible source of IL-22 in our model could be group 3 ILC. These cells might be contributing to the colitis in TRAG mice, but our finding that colitis occurs in TRAG $x$ Rorc $^{-/-}$ mice indicates that group 3 ILC are not required for the development of colitis in TRAG mice. Depletion of Thy $1.2^{+}$cells markedly reduced colitis in TRAG mice, suggesting that group 1 or 
a

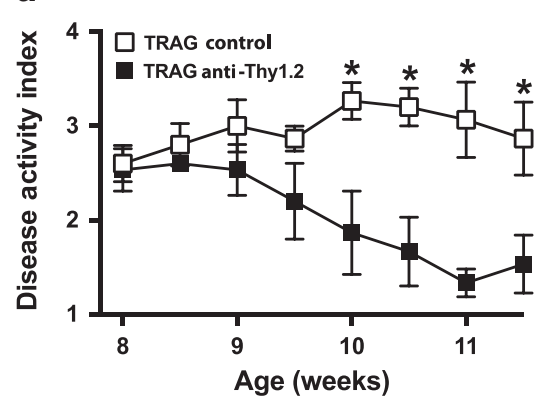

C

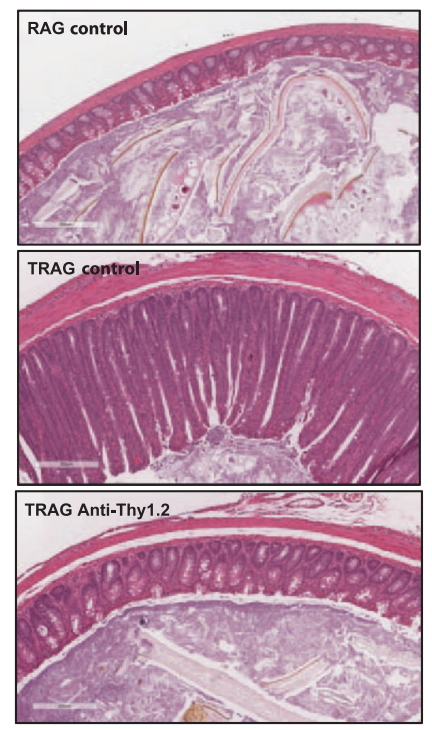

b

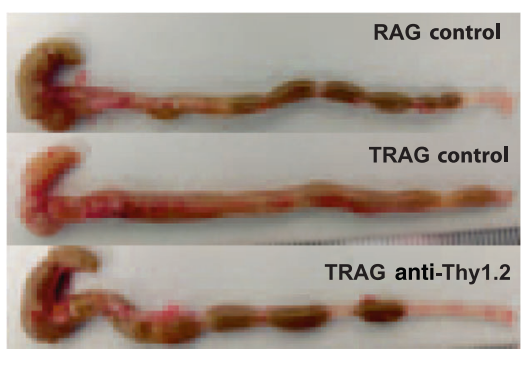

d

e
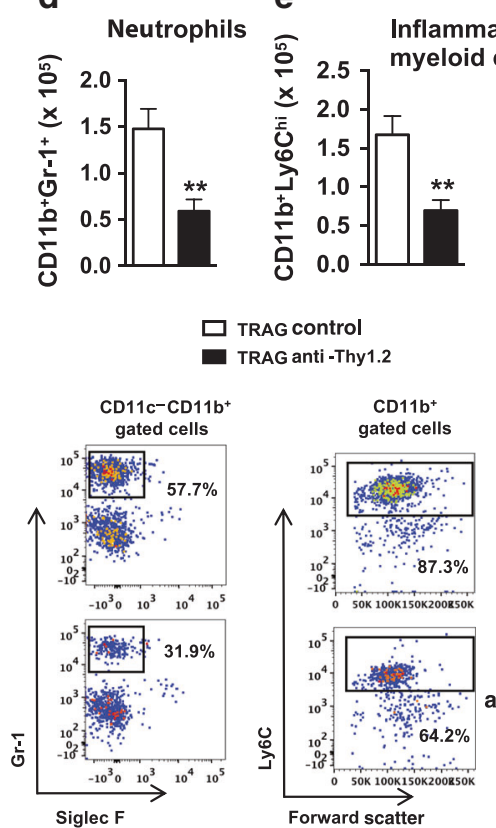

$\square$ TRAG control

TRAG anti -Thy1.2

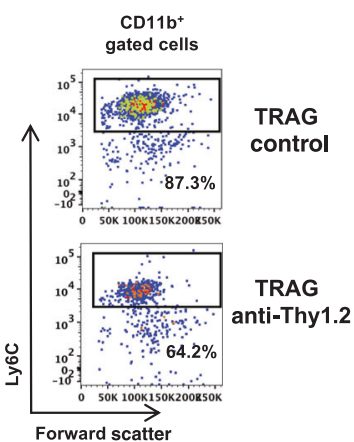

Fig. 5 Therapeutic anti-Thy1.2 treatment reverses established colitis in TRAG mice. Anti-Thy1.2 (1 mg/mouse; i.p.) treatments were administered every 3 days beginning at 8 weeks of age for 21 days. (a) Disease activity showing clinical improvement in Thy 1.2 treated TRAG mice. After treatment reduced colitis was evident by (b) gross morphology of the colon and (c) histology. Colonic LPLs were assessed for total numbers of (d) neutrophils and (e) inflammatory myeloid cells (for (a) two-way ANOVA with sidak multiple comparison, $T$-test for other data). ${ }^{*} p<0.05,{ }^{* *} p<0.01$. Data represent of two independent experiments, mean \pm SEM ( $n=5$ mice/group)

group 2 ILC contribute to the colitis in this model. Group 1 ILC (including ILC1 and NK cells) produce significant quantities of IFNY and TNF, which were both found to be increased in the supernatants of cultured LPLs from TRAG mice. Group 1 ILC have been implicated in mouse models of IBD and in human IBD and may be pathogenic in TRAG colitis. ${ }^{23,}{ }^{24}$ Group 2 ILC make Th2type cytokines and we observed increased levels of both IL-5 and IL-9 in the supernatants of TRAG LPLs. Although Th2 cytokines can drive colitis in mouse models and are implicated in human ulcerative colitis, ${ }^{25-27}$ the function of group 2 ILC in the intestine is considered primarily protective, defending against helminthes and mediating epithelial cell repair through production of amphiregulin. ${ }^{28,}{ }^{29}$ Indeed, we found that levels of amphiregulin were markedly increased in the transcriptome of TRAG mice, suggesting that group 2 ILC may have been actively working to repair damaged intestinal epithelium in the TRAG colitis model. Thus although we cannot exclude a role for group 2 ILC in this model, it is unlikely that they are acting as inducers of colitis in TRAG mice. Lastly, it is possible that the pathology of TRAG colitis involves more than one subset of ILC acting together to disrupt intestinal homeostasis. This is suggested by our finding that deletion of RORyt diminished but did not prevent colitis in TRAG mice. Further studies with mice lacking group 1, group 2, or combinations of groups of ILC will be necessary to resolve this question.

This is also the first study to demonstrate the efficacy of a JAK inhibitor in suppression of innate immune inflammation in the absence of adaptive immunity in vivo. JAK inhibitors are currently being developed or in clinical trials for IBD. ${ }^{15}$ These inhibitors work by blocking JAK-STAT signaling, which is a predominant mode of cell signaling by cytokine receptors. One concern about their use is that both pro- and anti-inflammatory cytokines signal through JAK-STAT pathways. ${ }^{30}$ Anti-inflammatory cytokines like IL10, and proinflammatory cytokines like IFNy exert their effects through JAK-STAT mediated suppression or induction of innate immune inflammation. Therefore it is not clear whether JAK inhibitors will suppress or enhance innate immune mediated inflammation. In vitro studies have been equivocal, with some studies showing suppression, whereas others show activation, of innate immune inflammation. ${ }^{17-20}$ Some of these discrepancies may be due to biphasic dose responses to JAK inhibition. ${ }^{30}$ In vivo studies will help to resolve some of these questions and, in the present study, we have shown that one JAK inhibitor, ruxolitinib, has beneficial effects in an innate immune-mediated colitis model. Thus, this JAK inhibitor suppressed, rather than activated, innate immune inflammation in vivo. De Wilde et al. ${ }^{31}$ demonstrated that the 
a

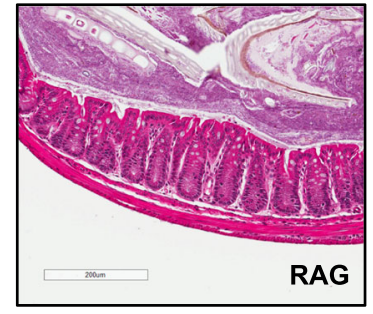

Antibiotic treatment of TRAG mice

b
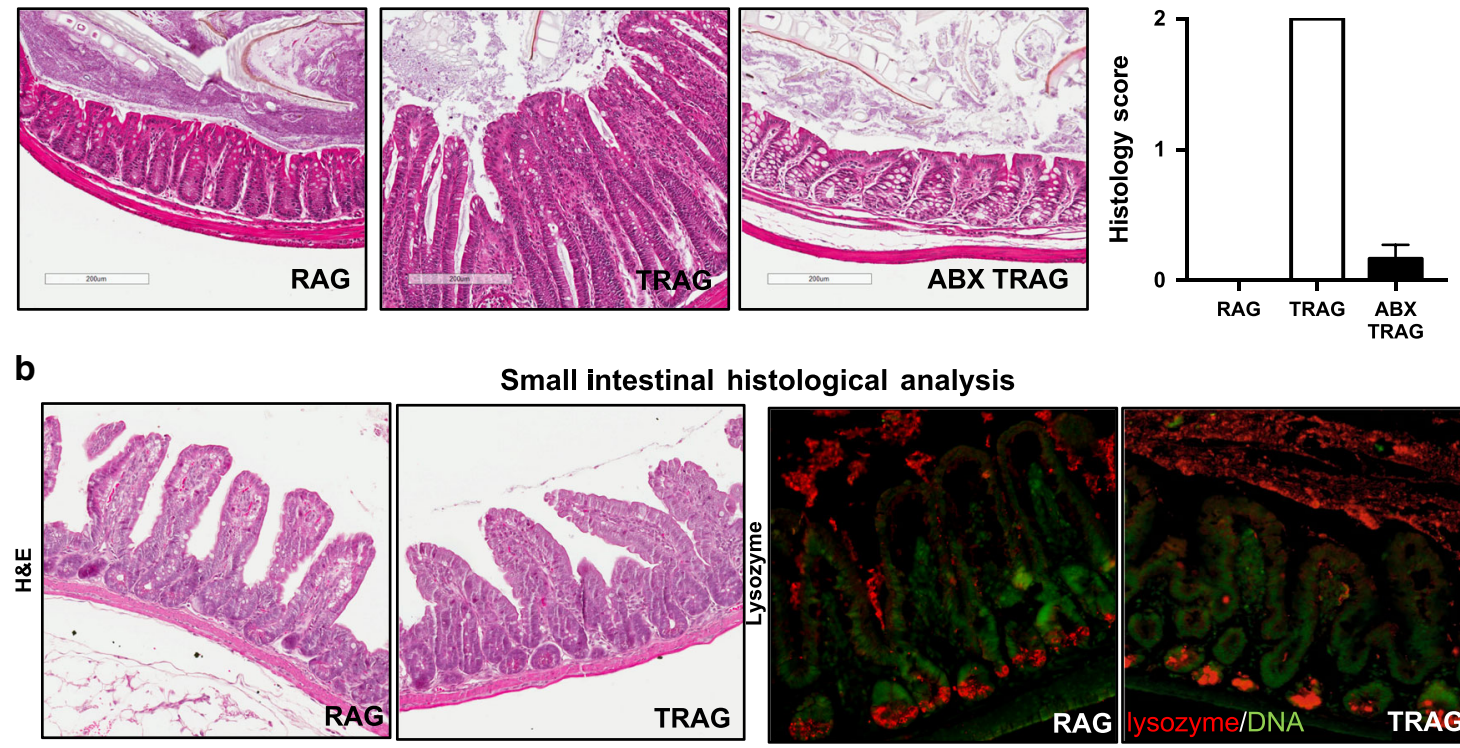

C

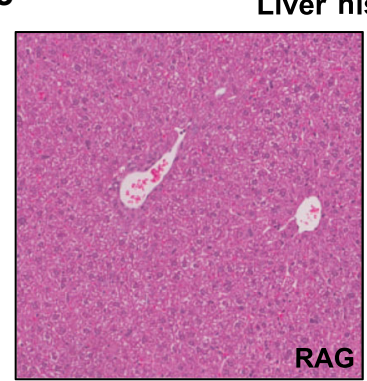

Small intestinal histological analysis

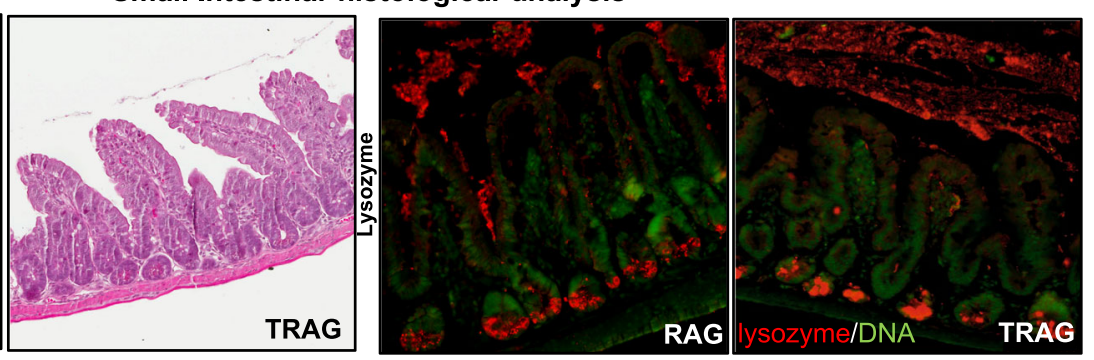

d

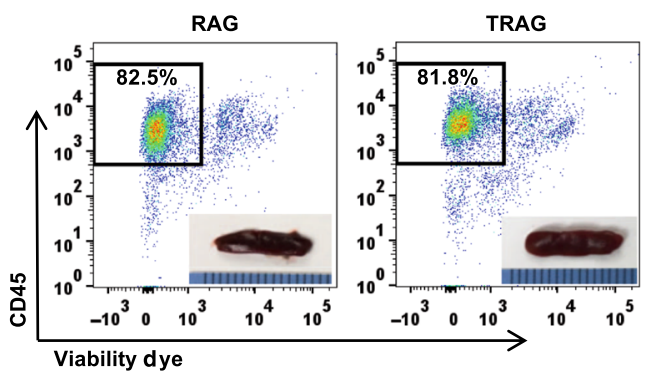

e

\section{ILC3 depletion of TRAG mice}
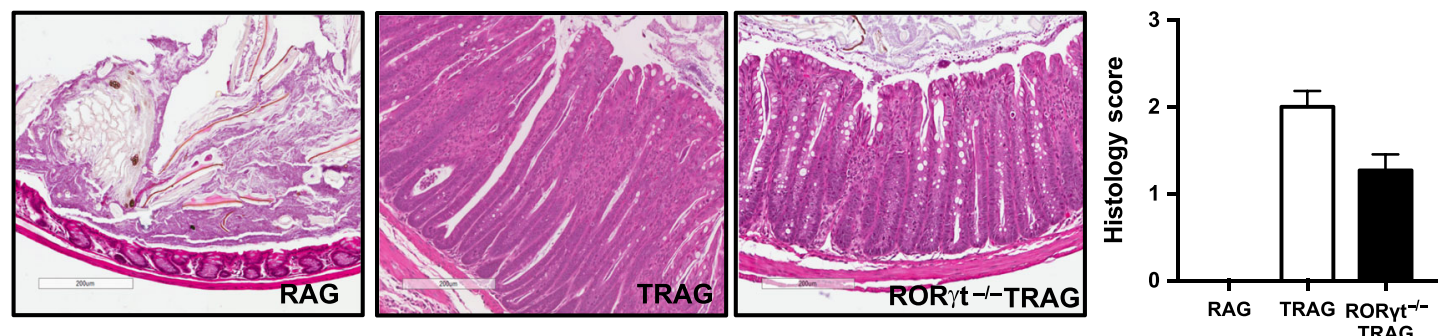

f
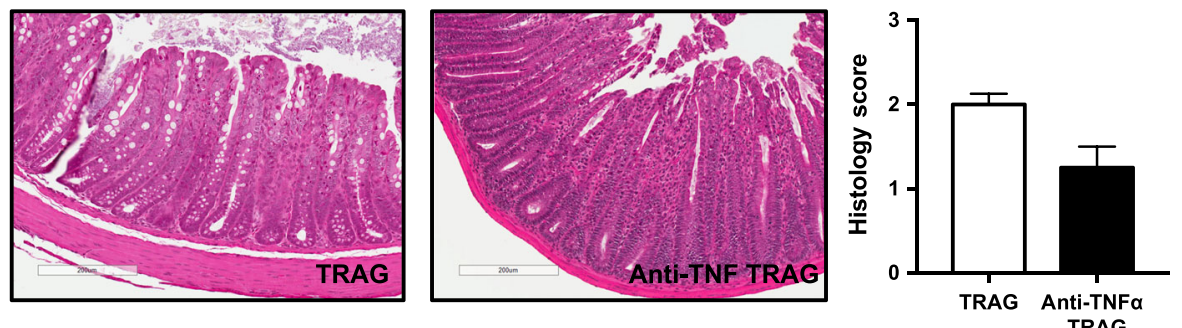

Fig. 6 TRAG colitis is microbially driven, restricted to the colon, and independent of group 3 ILCs. (a) Histology and histological cores showing suppression of colitis in antibiotic treated TRAG mice (ABX TRAG). Lack of small bowel and systemic inflammation in TRAG mice. (b) Histology showing absence of ileitis (upper panel H\&E) and preservation of Paneth cells (lower panel red =lysozyme) in the terminal ileum and (c) lack of hepatitis in liver of RAG1 $1^{-1-}$ or TRAG mice. (d) Flow cytometry and total cell counts of spleen showing lack of expansion of CD45 ${ }^{+}$ leukocytes in the primary immune tissue of TRAG mice (inset: picture of spleens). (e) Histology showing that colitis is not prevented in TRAG $x$ Rorc $^{-l-}$ mice and (f) in TRAG mice treated with anti-TNF antibody 

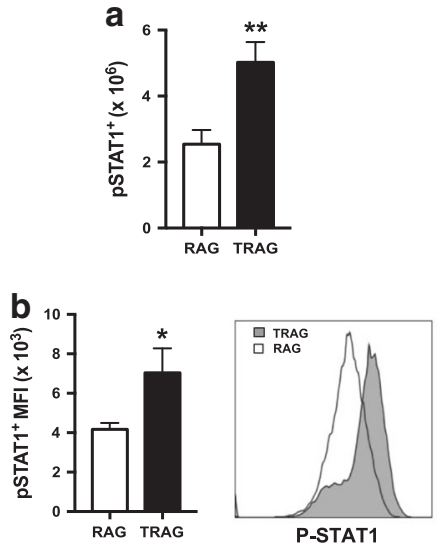

d

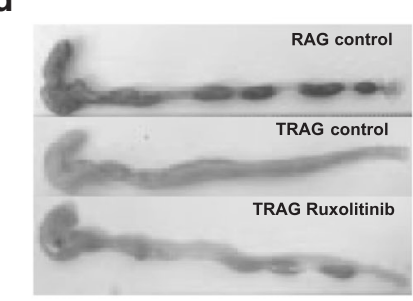

e

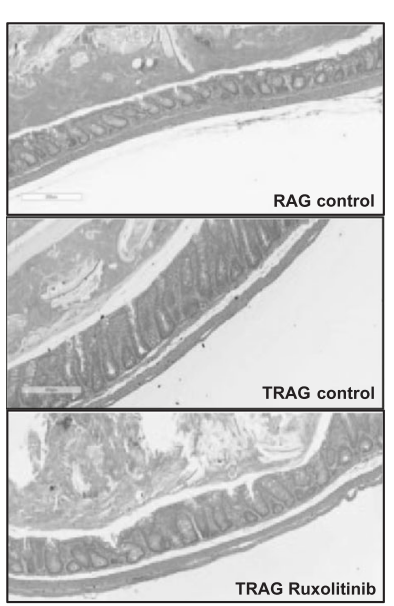

C

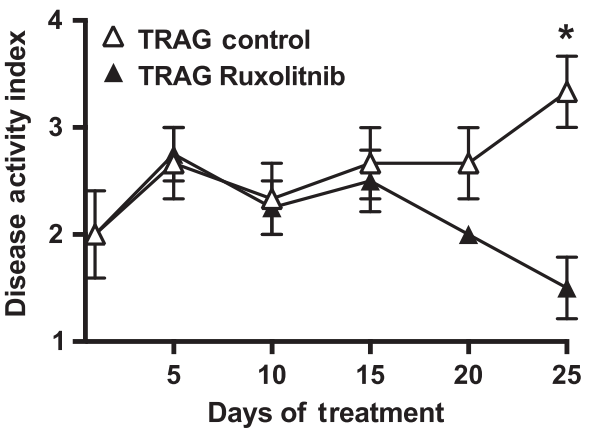

f
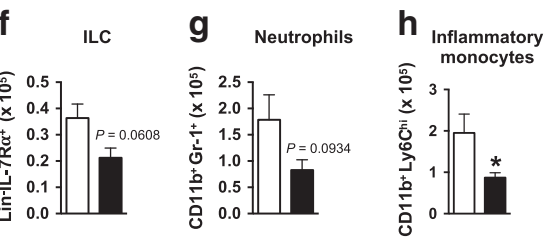

$\square$ TRAG control

TRAG Ruxolitinib
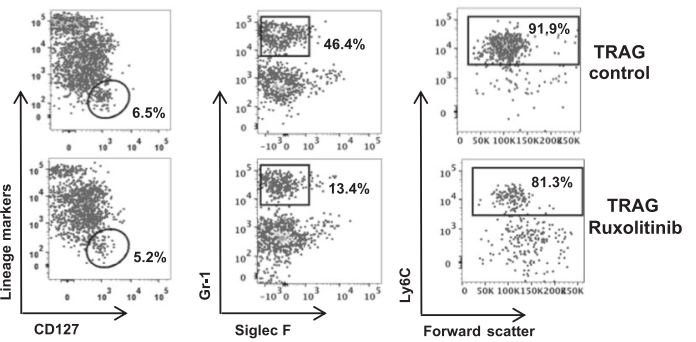

Fig. 7 The JAK1/2 inhibitor ruxolitinib reduced colitis in TRAG mice. Mice were gavaged twice daily $(60 \mathrm{mg} / \mathrm{kg})$ from 3 to 7 weeks of age. (a) Flow cytometry indicating that there are more phospho-STAT1 ${ }^{+}$Thy 1.2 cells that also (b) have a higher MFI for phospho-STAT1 in LPLs from TRAG mice compared to those of RAG $1^{-1-}$ mice. Clinical improvement following ruxolitinib treatment was indicated by (c) disease activity, (d) gross colon morphology, and (e) histology. (f-h) LPL counts show a significant reduction of inflammatory monocytes and a trend of decreased leukocytes, ILCs, and neutrophils in the colonic mucosa of ruxolitinib-treated mice (for (c) two-way ANOVA with Sidak multiple comparison test, $T$-test for other data) ${ }^{*} p<0.05, p<0.01$. Data represent mean \pm SEM ( $n=3-4$ mice/group)

JAK inhibitor tofacitinib prevented enthesitis caused by myeloid specific ablation of TNFAIP3 in vivo in an immune competent mouse model, indicating that JAK inhibition is a viable approach to treatment of inflammation initiated by innate immune cells. The cellular targets of JAK Inhibitors in our model have yet to be determined. We observed increased activation of JAK/STAT signaling in Thy $1.2^{+}$cells and amelioration of colitis in mice depleted of Thy $1.2^{+}$cells, suggesting that these could be one target of JAK inhibitors in prevention of inflammation. However, many other cell types, including myeloid cells and IECs are activated by cytokines that act through JAK/STAT signaling and inhibition of responses to cytokines in these cell types may also be contributing to reduction of inflammation by ruxolitinib in TRAG mice. It is possible that multiple cytokines acting in a concerted manner bring about the inflammation in TRAG mice and in human IBD. For this reason, the use of JAK inhibitors, which act on multiple cytokine pathways simultaneously, hold significant promise as IBD therapies. The robust and predictable nature of the colitis in TRAG mice should allow us to investigate whether ruxolitinib or other JAK inhibitors can also act therapeutically to induce remission in an innate model of colitis.

The TRAG colitis is distinct from previously described models of innate immune-mediated colitis. Mice lacking the transcription factor T-bet $\left(\mathrm{Tb} \times 21^{-/-}\right)$develop spontaneous colitis when crossed to RAG2 ${ }^{-1-}$ mice (TRUC mice) on a Balb/C background. ${ }^{32}$ The colitis in TRUC mice is transmissible to co-housed immunocompetent littermates, characterized by high levels of TNF and suppressed by anti-TNF therapy. ${ }^{32}$ Although we found that microbes drive colitis in TRAG mice, and that LPLs from TRAG mice secreted excess TNF we did not observe transmission of disease or amelioration with anti-TNF therapy. In addition, TRUC colitis is markedly attenuated on the C57BI/6 background, 
whereas TRAG colitis is robust and early onset in this background. ${ }^{33}$ Lastly, TRUC colitis is not characterized by a high IFNY cytokine profile, whereas TRAG colitis includes a high IFNY profile. $^{32}$ In addition to spontaneous innate models of inflammation there are induced innate models of colitis. These include antiCD40 treatment of $\mathrm{RAG}^{-1-}$ mice, which is accompanied by systemic inflammation and wasting disease, or $H$. hepaticus infection of immunodeficient mice (i.e., 129SvEvRAG ${ }^{-1-}$, SCID, or nude mice), accompanied by hepatitis and typhlitis, which are not seen in TRAG mice. ${ }^{34-37}$ We find that TRAG colitis is suppressed by ILC depletion and this is also the case in TRUC mice, and in induced models of innate colitis. ${ }^{38,39,4}$ Most importantly, we find that TRAG colitis is not prevented by deletion of RoRyt, suggesting that, unlike other innate colitis models, the colitis in TRAG mice is not dependent on group 3 ILC or cells derived from plasticity of group 3 ILC. Thus, although TRUC colitis or induced models of colitis may share some immunopathology with TRAG colitis, the colitis in TRAG mice is distinct and likely to provide unique insights into the regulation of intestinal inflammation. As our model is distinct but shares some features of other innate immune models of IBD it will be of interest to compare these models. For example, regulatory T cells can both prevent and cure colitis in the $H$. hepaticus model of innate IBD, which is driven by IL-23 and group 3 ILCs. ${ }^{4}, 35,40,41$ Whether regulatory T cells have this effect on ILC3-independent colitis in TRAG mice, will provide new insight into possible regulation of inflammation by regulatory $T$ cells.

$\mathrm{TNFAIP}^{-/-} \times \mathrm{RAG}^{-/-}$mice develop spontaneous colitis owing to a critical role for TNFAIP3 in suppressing myeloid cell responses to microbial stimulation. ${ }^{5,7,10}$ This colitis is distinct from TRAG colitis, as TNFAIP3 ${ }^{-1-} \times \mathrm{RAG}^{-1-}$ mice display widespread systemic inflammation including inflammation in the liver, lung, skin, spleen and kidneys, which is not seen in TRAG colitis. ${ }^{7}$ Given the critical anti-inflammatory role of TNFAIP3 it is interesting that transgenic expression of TNFAIP3 in IEC predisposes to colitis. This likely relates to cell and context dependent roles for TNFAIP3, similar to the cell and context dependent roles of nuclear factor (NF)- $\mathrm{KB}$, a transcription factor that is negatively regulated by TNFAIP3. ${ }^{5,7}$ Activation of NF-KB in innate immune cells is required for inflammation, but loss of NF-KB activation in IEC leads to spontaneous intestinal inflammation owing to a critical role of NF$K B$ in protection of IEC from cytokine-induced death. ${ }^{42,43}$ Similarly, TNFAIP3, which inhibits NF-KB activation, may have cell context dependent roles in colitis. TNFAIP3 protects against DSS-induced IEC death, ${ }^{12}$ but in TRAG colitis ulceration and denudation of the epithelium is occasionally evident, suggestive of IEC death in this model. Thus TNFAIP3 expression in IEC may increase colitis susceptibility through mechanisms similar to those observed in conditions where NF-KB activation in IEC is blocked, namely increased epithelial cell susceptibility to cytokine-induced death. ${ }^{42,43}$

One explanation for colitis in TRAG mice may be that expression of TNFAIP3 in IEC changes the biogeography of the intestinal microbiome, thus predisposing to colitis in immunodeficient hosts. ${ }^{14}$ In normal unchallenged mice, the colonic epithelium is covered by an inner layer of mucus that is relatively devoid of microbes. ${ }^{44}$ In villin-TNFAIP3 mice this inner mucus layer is invaded by microbes. ${ }^{14}$ Despite this, villin-TNFAIP3 mice never spontaneously develop colitis but instead display increased numbers of mucosal Tregs, which may prevent spontaneous inflammation. ${ }^{14}$ Indeed, villin-TNFAIP3 $\times \mathrm{IL}^{-1} 0^{-1}$ mice exhibit invasion of the inner mucus layer by microbes and develop $100 \%$ penetrant early-onset colitis on the $\mathrm{C} 57 \mathrm{BI} / 6$ background that is not observed in IL $10^{-1-}$ or villin-TNFAIP3 mice. ${ }^{14}$ Thus, the combination of microbial invasion of the inner mucus layer and immunodeficiency (IL10 deficiency) results in colitis. It is likely that a similar process of altered microbial biogeography and immunodeficiency results in colitis in TRAG mice. As we have observed that microbes drive colitis in TRAG mice, it will be of interest to determine how microbes interact with the intestinal mucosa to induce ILC, inflammatory cytokines, and activation of JAK/STAT signals to promote inflammation.

\section{MATERIALS AND METHODS}

Mice

Mice were bred and housed in Freimann Life Sciences Center at the University of Notre Dame. Previously, these mice were generated, bred and housed at the University of Chicago animal care facility. All experiments were approved by Institutional Animal Care and Use Committees of both institutions. All mice were bred and maintained on the C57BL/6 background. The villin-TNFAIP3 strain was generated previously using BAC-recombineering of the villin locus and characterized as described. ${ }^{13} \mathrm{RAG}^{-/-}$mice (C57BI/ 6) were purchased from Jackson Laboratories and interbred to villin-TNFAIP3 mice to generate villin-TNFAIP3 $\times \mathrm{RAG}^{-1-}$ (TRAG) mice or RAG $1^{-/-}$littermate controls. To generate TRAG $\times$Rorc $^{-/-}$ mice, Rorc $^{+-}$mice were purchased from Jackson labs, interbred with RAG1 ${ }^{-1-}$ mice, and then crossed to villin-TNFAIP3 mice to generate TRAG $\times$ Rorc $^{-/-}$mice or TRAG Rorc ${ }^{+/-}$littermate controls.

\section{Assessment of colitis}

Mice were monitored either bi-weekly or at each treatment point and disease activity scores were assigned. Disease activity scores were averaged based on three categories, weight loss, blood in stool, and stool consistency. For weight, no weight loss was scored as 1 , weight loss of $1-5 \%$ from baseline as $2,5-10 \%$ as $3,10-15 \%$ as 4 , and $>15 \%$ as 5 . Hemoccult test strips and direct visualization were used to assess blood in stool with no blood scored as 1, blood-positive stool scored as 3, and overt rectal bleeding scored as 5. Stool consistency was monitored as normal consistency scored as 1, pasty or semiformed stool scored as 3, and liquid stool or diarrhea scored as 5. At necropsy the colon and cecum were excised and measured for length. Tissues including colon, small intestine, and liver were collected and formalin-fixed and paraffinembedded tissues were sectioned $(4-5 \mu \mathrm{m})$ and stained by hematoxylin and eosin for pathological scoring or immunohistochemistry. Histological scores for colitis were assigned by a board certified gastrointestinal pathologist (C.R.W.) blinded to genotypes and treatments using the criteria: $0=$ histologically normal; $1=$ intraepithelial neutrophils (mild activity) also associated with karyorectic debris and proliferation; $2=$ crypt abscesses; $3=>50 \%$ crypt abscesses; $4=$ epithelial erosions or ulcers.

\section{LPL extraction and flow cytometry}

Colons along with cecum (without cecal lymphoid patch) were excised, thoroughly cleared of fecal content and residual fat, and cut longitudinally and into $2 \mathrm{~cm}$ sections. The sections were washed with several rounds of PBS to remove the remaining feces. To eliminate IECs, tissue sections were shaken for $\sim 2 \mathrm{~h}$ at $37^{\circ} \mathrm{C}$ in RPMI with $1 \mathrm{mM}$ of EDTA. Shaking for an additional $\sim 2 \mathrm{~h}$ with collagenase (Sigma) and DNase (Sigma) in RPMI to isolate the LPLs. Viable nucleated cells were counted and used for flow cytometry analysis.

\section{Flow cytometry}

LPL single-cell suspensions, or other leukocytes where indicated, were incubated with anti-CD16/32 (clone: 93) (eBioscience, San Diego, CA) to reduce nonspecific binding. Cells were incubated on ice for 30 mins in the dark, with a combination of fluorescent antibodies (eBioscience). A cocktail consisting of anti-CD127 PE (clone: A7R34), anti-CD45.2 PE-eFluor 610 (104), anti-CD3 eFluor 450 (17A2), anti-CD19 eFluor 450 (eBio1D3), anti-NK1.1 eFluor 450 (PK136), anti-Gr-1 efluor 450 (RB6-8C5), anti-Ter119 efluor 450 (TER-119), and anti-CD11b eFluor $450(\mathrm{M} 1 / 70)$, or anti-CD45.2 PerCP-Cy5.5 (104), anti-CD11b PE (M1/70), anti-Gr-1 eFluor 450 
(RB6-8C5), anti-Siglec F eFluor 660 (1RNM44N), anti-Ly6C PE-Cy7 (HK1.4). Stained cells were incubated with a fixable viability dye (eBioscience), washed, and subsequently fixed with $2 \%$ paraformaldehyde. Samples were acquired using a BD LSRFortessa X-20 (BD Biosciences, San Jose, CA) and analyzed on FlowJo v10 software (Tree Star, Inc., Ashland, OR).

\section{RNA isolation and analysis}

Immediately after colon extraction, a small section of the distal colon $\sim 2 \mathrm{~mm}$ was snap frozen, homogenized with Trizol (Life Technologies), isolated with chloroform, isopropanol, and ethanol, and suspended in RNase-free water as per manufacturer's protocol. For total transcriptome analysis, RNA samples were analyzed with the Illumina NextSeq500 with libraries constructed using the Illumina TruSeq Standard mRNA kit. Overall, $98.5 \%$ of the reads passed the quality filters and trimming, which incorporated quality filtering (q20) and allowing a minimum read length of 30 bases. Cleaned reads were than mapped to Mouse genome (Mus musculus ver. GRCm38.75) using Tophat ver. 2.1.0. Differential Expression analysis was conducted using Deseq2. Sequence expressions were normalized and Wald's $X^{2}$-tests were conducted on each gene to compare TRAG vs. RAG $1^{-/-}$gene total colonic tissue gene expression. Ingenuity Pathway Analysis software was utilized for genes expression profiles and pathways analysis.

For reverse transcription-PCR (RT-PCR), RNA was isolated from the distal colon using Trizol (Life Technologies) and CDNA was generated using High Capacity cDNA Reverse Transcription Kits (Applied Biosystems) following manufacturer's instructions. Quantitative PCR was performed with SYBR Green (Qiagen) in duplicate with two control housekeeping genes, 18S, and actin. The primer pairs (Integrated DNA Technologies) used for gene amplification were as follows: IFN- $\square$ fwr: 5'-CCATCAGCAACAACATAAGC-3', rev: 5'-AGCTCATTGAATGCTTGGCG-3'; IL-22 fwr: 5'-CGCTGCCCGTCAA CACCCGG-3', rev: 5'-CTGATCCTTAGCACTGACTCCTCG-3'; IL-17f fwr: 5'-AACCAGGGCATTTCTGTCCC-3', rev: 5'-TाTCTTGCTGAATGGCG ACG-3'. Amplicon intensity was captured by Applied Biosystems StepOne Plus RT-PCR machine. CT values were normalized using the housekeeping genes and to compare gene expression in TRAG vs. $\mathrm{RAG}^{-1-}$ colon tissue.

Cytokine secretion from LPLs

Freshly isolated LPLs $\left(10^{6}\right)$ isolated from intestinal mucosa were incubated overnight $(18 \mathrm{~h})$ in complete RPMI with $10 \%$ fetal calf serum without additional stimulation. Supernatants were assessed for cytokine levels with the Biolegend LEGENDplex cytokine bead array following manufacturers instructions (Biolegend, San Diego CA). Cytokines assayed in this array included: IL-23, IL-5, IFNY, TNF, IL-12 (p70), IL-4, IL-1 $\beta$, GM-CSF, IL-9, IL-17F, IL-17A, IL-22, and IL-13.

\section{Antibody-based depletions in vivo}

Innate lymphoid cells. Anti-Thy1.2 (IgG2b) antibody was generated from rat hybridoma cells (ATCC $30-\mathrm{H} 12$ ). The hybridoma cells were grown in RPMI-1640 (ATCC) with $10 \%$ fetal bovine serum and passaged at $30-40 \%$ confluence every other day. To extract the antibody, cells were grown to high density and switched to serum-free media for 2 days before the supernatant containing the antibody was collected, filtered, and concentrated using Vivaspin 5000 Protein Concentrator Spin Columns (GE Healthcare Life Sciences). HiTrap Protein G HP column (GE Healthcare Life Sciences) was then utilized to isolate the $\lg$ antibody. The concentration of anti-Thy 1.2 was measured using a rat $\operatorname{lgG} 2$ Elisa kit (eBiosciences). Anti-Thy 1.2 antibody was injected $(1 \mathrm{mg} / \mathrm{mouse}$; i.p.) every 3 days, with controls receiving pre-immune rat serum. Two different treatment strategies with Thy 1.2 were conducted. The first focused on colitis prevention, mice received the treatment at 4 weeks of age, received 5 doses, and were killed at 6-7 weeks of age. The second treatment strategy was geared toward colitis treatment, mice at 8 weeks of age with already established inflammation were given eight treatments and killed at 11-12 weeks of age. Mice were monitored and disease activity scores were administered at each treatment point.

Tumor necrosis factor. Four-week-old mice were treated with anti-TNF antibody (TN3-19.12) or control serum at a dose of 15ug/ $\mathrm{g}$ every 7 days for 4 weeks, as described. ${ }^{32}$

Antibiotic treatments

Weanlings were maintained on a cocktail of antibiotics dissolved in drinking water ad libitum until 6 weeks of age. The antibiotic cocktail was comprised of vancomycin $(500 \mathrm{mg} / \mathrm{L})$, neomycin sulfate $(1 \mathrm{~g} / \mathrm{L})$, metronidazole $(1 \mathrm{~g} / \mathrm{L})$, and ampicillin $(1 \mathrm{~g} / \mathrm{L})$, and was dissolved in grape Kool-Aid to facilitate consumption. Control mice received Kool-Aid without antibiotics.

Inhibition of JAK in vivo

Mice received Ruxolitinib (AdoQ INB018424) via gavage at $60 \mathrm{mg} /$ $\mathrm{kg}$ in DMSO, or vehicle control, twice daily from 3 to 7 weeks of age. Mice were assessed for clinical signs of colitis and assessed at euthanasia as described.

Statistics

Statistical analysis was performed using IBM SPSS and GraphPad Prism software using analysis of variance (ANOVA) with post hoc tests. For clinical scores data was analyzed by two-way ANOVA with Sidak multiple comparisons test. Mann-Whitney tests or Student's $T$-tests were performed for paired data, where indicated. Significance was inferred at $p<0.05$.

\section{ACKNOWLEDGEMENTS}

This work was supported by NIH support from NIAID (AI083375-01), NIDDK (DK42086), and the Crohn's and Colitis Foundation of America 0-34493-1362

\section{AUTHOR CONTRIBUTIONS}

A.M.O., D.L.T., and L.A.C. designed and performed experiments, analyzed data, and contributed to writing the manuscript. S.F.M., L.R., A.M.O., A.M.B., K.R.A., A.M.I., and D. S.B. performed experiments and reviewed the manuscript. C.R.W. scored and analyzed data. D.L.B. conceived the project, designed experiments, and wrote the manuscript.

\section{ADDITIONAL INFORMATION}

Conflict of interest: The authors declare no competing interest.

Publisher's note: Springer Nature remains neutral with regard to jurisdictional claims in published maps and institutional affiliations.

\section{REFERENCES}

1. Strober, W., Fuss, I. J. \& Blumberg, R. S. The immunology of mucosal models of inflammation. Annu. Rev. Immunol. 20, 495-549 (2002).

2. Artis, D. \& Spits, H. The biology of innate lymphoid cells. Nature 517, 293-301 (2015).

3. Geremia, A. et al. IL-23-responsive innate lymphoid cells are increased in inflammatory bowel disease. J. Exp. Med. 208, 1127-33 (2011).

4. Buonocore, S. et al. Innate lymphoid cells drive interleukin-23-dependent innate intestinal pathology. Nature 464, 1371-5 (2010).

5. Boone, D. L. et al. The ubiquitin-modifying enzyme A20 is required for termination of Toll-like receptor responses. Nat. Immunol. 5, 1052-60 (2004).

6. Wertz, I. E. et al. De-ubiquitination and ubiquitin ligase domains of A20 downregulate NF-kappaB signalling. Nature 430, 694-9 (2004).

7. Lee, E. G. et al. Failure to regulate TNF-induced NF-kappaB and cell death responses in A20-deficient mice. Science 289, 2350-4 (2000).

8. Ma, A. \& Malynn, B. A. A20: linking a complex regulator of ubiquitylation to immunity and human disease. Nat. Rev. Immunol. 12, 774-85 (2012).

9. Jostins, L. et al. Host-microbe interactions have shaped the genetic architecture of inflammatory bowel disease. Nature 491, 119-24 (2012). 
10. Turer, E. E. et al. Homeostatic MyD88-dependent signals cause lethal inflamMation in the absence of A20. J. Exp. Med. 205, 451-64 (2008).

11. Vereecke, L. et al. A20 controls intestinal homeostasis through cell-specific activities. Nat. Commun. 5, 5103 (2014).

12. Rhee, L. et al. Expression of TNFAIP3 in intestinal epithelial cells protects from DSS- but not TNBS-induced colitis. Am. J. Physiol. Gastrointest. Liver Physiol. 303, G220-227 (2012)

13. Kolodziej, L. E. et al. TNFAIP3 maintains intestinal barrier function and supports epithelial cell tight junctions. PLoS ONE 6, e26352 (2011).

14. Murphy, S. F. et al. Intestinal epithelial expression of TNFAIP3 results in microbial invasion of the inner mucus layer and induces colitis in IL10-deficient mice. Am. J. Physiol. Gastrointest. Liver Physiol. 307, G871-882 (2014).

15. Neurath, M. F. Current and emerging therapeutic targets for IBD. Nat. Rev. Gastroenterol. Hepatol. 14, 269-78 (2017).

16. Barrett, J. C. et al. Genome-wide association defines more than 30 distinct susceptibility loci for Crohn's disease. Nat. Genet. 40, 955-62 (2008).

17. Ghoreschi, K. et al. Modulation of innate and adaptive immune responses by tofacitinib (CP-690,550). J. Immunol. 186, 4234-43 (2011).

18. Yarilina, A., Xu, K., Chan, C. \& Ivashkiv, L. B. Regulation of inflammatory responses in tumor necrosis factor-activated and rheumatoid arthritis synovial macrophages by JAK inhibitors. Arthritis Rheum. 64, 3856-66 (2012).

19. Pattison, M. J., Mackenzie, K. F. \& Arthur, J. S. Inhibition of JAKs in macrophages increases lipopolysaccharide-induced cytokine production by blocking IL-10mediated feedback. J. Immunol. 189, 2784-92 (2012).

20. Wang, $H$. et al. The role of JAK-3 in regulating TLR-mediated inflammatory cytokine production in innate immune cells. J. Immunol. 191, 1164-74 (2013).

21. Santos, N. C., Figueira-Coelho, J., Martins-Silva, J. \& Saldanha, C. Multidisciplinary utilization of dimethyl sulfoxide: pharmacological, cellular, and molecular aspects. Biochem. Pharmacol. 65, 1035-41 (2003).

22. Kirchberger, S. et al. Innate lymphoid cells sustain colon cancer through production of interleukin-22 in a mouse model. J. Exp. Med. 210, 917-31 (2013).

23. Bernink, J. H. et al. Human type 1 innate lymphoid cells accumulate in inflamed mucosal tissues. Nat. Immunol. 14, 221-9 (2013).

24. Fuchs, A. et al. Intraepithelial type 1 innate lymphoid cells are a unique subset of IL-12- and IL-15-responsive IFN-gamma-producing cells. Immunity 38, 769-81 (2013).

25. Fuss, I. J. et al. Disparate CD4 + lamina propria (LP) lymphokine secretion profiles in inflammatory bowel disease. Crohn's disease LP cells manifest increased secretion of IFN-gamma, whereas ulcerative colitis LP cells manifest increased secretion of IL-5. J. Immunol. 157, 1261-70 (1996).

26. Strober, W. \& Fuss, I. J. Proinflammatory cytokines in the pathogenesis of inflammatory bowel diseases. Gastroenterology 140, 1756-67 (2011).

27. Rosen, M. J. et al. Mucosal expression of type 2 and type 17 immune response genes distinguishes ulcerative colitis from colon-only Crohn's disease in treatment-naive pediatric patients. Gastroenterology 152, 1345-57 e1347 (2017).
28. Monticelli, L. A. et al. IL-33 promotes an innate immune pathway of intestinal tissue protection dependent on amphiregulin-EGFR interactions. Proc. Natl Acad. Sci. USA 112, 10762-7 (2015).

29. von Moltke, J., Ji, M., Liang, H. E. \& Locksley, R. M. Tuft-cell-derived IL-25 regulates an intestinal ILC2-epithelial response circuit. Nature 529, 221-5 (2016).

30. Hedl, M., Proctor, D. D. \& Abraham, C. JAK2 disease-risk variants are gain of function and JAK signaling threshold determines innate receptor-induced proinflammatory cytokine secretion in macrophages. J Immunol 197, 3695-704 (2016).

31. De Wilde, K. et al. A20 inhibition of STAT1 expression in myeloid cells: a novel endogenous regulatory mechanism preventing development of enthesitis. Ann. Rheum. Dis. 76, 585-92 (2017).

32. Garrett, W. S. et al. Communicable ulcerative colitis induced by T-bet deficiency in the innate immune system. Cell 131, 33-45 (2007).

33. Ermann, J. et al. Severity of innate immune-mediated colitis is controlled by the cytokine deficiency-induced colitis susceptibility-1 (Cdcs1) locus. Proc. Natl Acad. Sci. USA 108, 7137-41 (2011).

34. Li, X. et al. SCID/NCr mice naturally infected with Helicobacter hepaticus develop progressive hepatitis, proliferative typhlitis, and colitis. Infect. Immun. 66, 5477-84 (1998).

35. Maloy, K. J. et al. $C D 4+C D 25+T(R)$ cells suppress innate immune pathology through cytokine-dependent mechanisms. J. Exp. Med. 197, 111-9 (2003).

36. Ward, J. M. et al. Inflammatory large bowel disease in immunodeficient mice naturally infected with Helicobacter hepaticus. Lab. Anim. Sci. 46, 15-20 (1996).

37. Uhlig, H. H. et al. Differential activity of IL-12 and IL-23 in mucosal and systemic innate immune pathology. Immunity 25, 309-18 (2006).

38. Ermann, J., Staton, T., Glickman, J. N., de Waal Malefyt, R. \& Glimcher, L. H. Nod/ Ripk2 signaling in dendritic cells activates IL-17A-secreting innate lymphoid cells and drives colitis in T-bet-/-.Rag2-/- (TRUC) mice. Proc. Natl Acad. Sci. USA 111, E2559-2566 (2014)

39. Powell, N. et al. The transcription factor T-bet regulates intestinal inflammation mediated by interleukin-7 receptor + innate lymphoid cells. Immunity $37,674-84$ (2012).

40. Kullberg, M. C. et al. IL-23 plays a key role in Helicobacter hepaticus-induced T celldependent colitis. J. Exp. Med. 203, 2485-94 (2006).

41. Maloy, K. J., Antonelli, L. R., Lefevre, M. \& Powrie, F. Cure of innate intestinal immune pathology by $\mathrm{CD} 4+\mathrm{CD} 25+$ regulatory T cells. Immunol. Lett. 97, 189-92 (2005).

42. Zaph, C. et al. Epithelial-cell-intrinsic IKK-beta expression regulates intestinal immune homeostasis. Nature 446, 552-6 (2007).

43. Nenci, A. et al. Epithelial NEMO links innate immunity to chronic intestinal inflammation. Nature 446, 557-61 (2007).

44. Hansson, G. C. \& Johansson, M. E. The inner of the two Muc2 mucin-dependent mucus layers in colon is devoid of bacteria. Gut Microbes 1, 51-54 (2010). 